\title{
Media Synchronization Workshop
}

$\begin{array}{ll}\text { Hans Stokking } & \begin{array}{l}\text { Fernando Boronat } \\ \text { TNO }\end{array} \\ \begin{array}{l}\text { UPV } \\ \text { The Netherlands } \\ \text { hans.stokking@tno.nl }\end{array} & \begin{array}{l}\text { Spain } \\ \text { fboronat@dcom.upv.es }\end{array} \\ & \\ \text { Pablo Cesar } & \text { Mario Montagud } \\ \text { CWI } & \text { CWI } \\ \text { The Netherlands } & \text { The Netherlands } \\ \text { p.s.cesar@cwi.nl } & \text { M.Montagud@cwi.nl }\end{array}$

Permission to make digital or hard copies of part or all of this work for personal or classroom use is granted without fee provided that copies are not made or distributed for profit or commercial advantage and that copies bear this notice and the full citation on the first page. Copyrights for third-party components of this work must be honored. For all other uses, contact the owner/author(s). Copyright is held by the author/owner(s)

$-5,2015$, Brussels, Belgium

(26-3/15/06.

http://dx.doi.org/10.1145/2745197.2745699

\begin{abstract}
The Media Synchronization workshop, in its third edition, brings together an active community around the topic of media synchronization, attracting relevant researchers in this area. The objective of the workshop is to further built this community and set the research agenda on the topic of media synchronization. We will do this by sharing our current research in short presentations, and by having an active session in the afternoon. We will be working in subgroups on key problem areas, present our work to the whole group as a starting point for an active discussion on the most relevant research to be carried out in the coming years.
\end{abstract}

\section{Author Keywords}

Media synchronization; distributed systems; media delivery; standards

\section{ACM Classification Keywords}

H.5.1 [Information interfaces and presentation]: Multimedia Information Systems

\section{Introduction}

The main objective of our workshop series is to create an active community around the topic of media synchronization. In this community we can share ideas and research agendas, we can collaborate in new projects, and bring new solutions to the industry. The goal in the workshops themselves is mainly to discuss the research agenda, to help us focus our work for the 
coming years. The lively discussions in the previous workshop editions have really helped set the agenda, both inside our community but also in the industry.

We have organized two previous editions of this workshop series [1], in 2012 and 2013. Both workshops attracted about 30 people: from European projects and national projects, from standardization, from the conference we co-located it with, and from our own contacts. The two previous editions had the format of presentations, with a long (panel) discussion at the end. The discussions were mostly about future research needed in the media synchronization domain. We now will run the third edition at the ACM International Conference on Interactive Experiences for TV and Online Video (ACM TVX2015), with a more interactive afternoon session. The goal is still to discuss relevant and emerging research challenges in the area of media synchronization.

\section{Background}

Media synchronization has been a challenge in A/V transmission for quite some time. Over the years, many techniques to achieve intra- and inter-media synchronization in various network conditions have been developed. In many cases, synchronization between different media is crucial for a satisfying perceived Quality of Experience (QoE). Currently, many new media synchronization issues arise. On the one hand, novel media technologies such as HTTP streaming protocols, media encoders and HDTV often require new synchronization techniques. On the other hand, new patterns in media consumption often introduce specific synchronization issues. For example, internet applications evolving around broadcast TV content may need synchronization between the application and the broadband stream. Synchronization between different TV receivers may be needed in Social TV (inter-destination media synchronization).

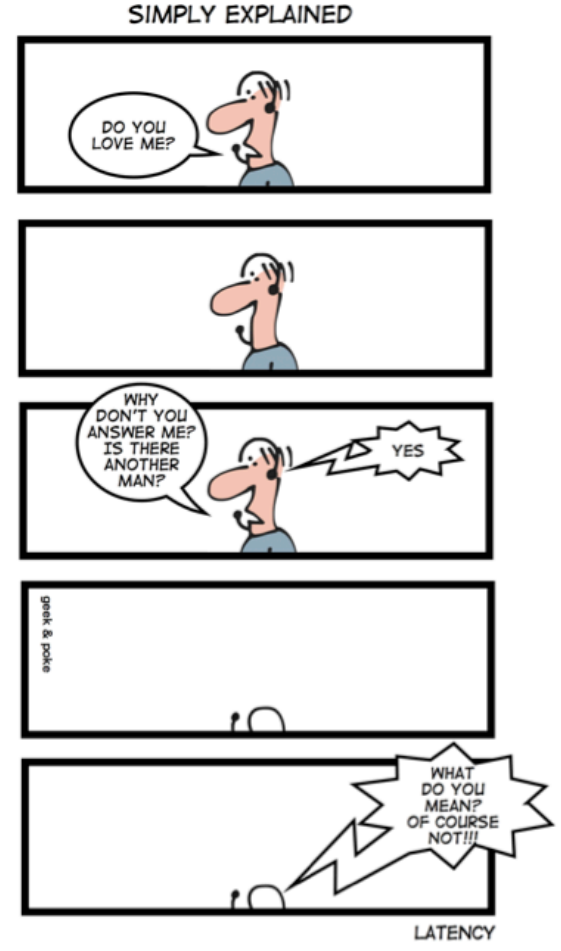

Geek and Poke, Simply Explained [2]

In some interactive TV cases synchronization between handheld devices and the TV screen may be needed. Moreover, novel 3D technologies for TV broadcasting and tele-presence (e.g., 3D tele-immersion) may require the adoption of several of these synchronization 
techniques to achieve a satisfying quality of user experience (QoE). Additionally, the deployment of applications that integrate, and synchronize inputs across all senses including the tactile, olfaction, and gustatory senses, apart from inputs for audio and visual senses, is currently a reality. The integration of such multiple sensorial effects into multimedia applications, or mulsemedia (multiple sensorial media) applications, also requires research on new media synchronization techniques, aligning rich data from multiple human senses, to provide a truly enriched and immersive experience. This workshop proposal addresses all the above synchronization issues and requests contributions from different perspectives.

\section{Scope and Goal}

The scope of the workshop is media synchronization in all its facets: architecture, protocols, algorithms, simulations, implementations, standardization, user experience studies, business models, etc. The goal of the workshop is to determine the most relevant research aspects of media synchronization in the coming years. Much research has been done in this area, as said, but many researchers involved in media synchronization have the feeling that they have not finished their job just yet. In this workshop, we want to concretely identify the most relevant research topics in the area of media synchronization that will require our effort in the coming years.

\section{Format}

We will follow an interactive format, using a subgroup working structure. From the submissions, we will determine the 3 most relevant topics for future research. On these topics, we will define short cases. These cases may be use cases, they may be major technical problem areas, they may be user experience issues, etc. We will divide the participants into 3 subgroups, and assign each of the subgroups one of the cases. The subgroups will be asked to work out the case, and provide a solution direction, or a clear insight into the problem. We will ask the subgroups to make 1 poster each on their work, which they will present to the whole group afterwards. These posters will be the input for a group discussion on the future of media synchronization research.

\section{Program}

The Media Synchronization Workshop 2015

(https://sites.google.com/site/mediasynchronization/m ediasync2015) will be held in conjunction with the ACM TVX on June 3rd, 2015, in Brussels, Belgium. The most pertinent contributions have been selected for inclusion in the workshop's agenda. These include seven papers:

1. A Test Bed for Hybrid Broadcast Broadband Services

2. An Automatic Media Synchronizing Mechanism with TV Programs

3. Leveraging Audio Fingerprinting for Hybrid Content Radio Synchronization

4. Multi-device Linear Composition on the Web, Enabling Multi-device Linear Media with HTMLTimingObject and Shared Motion

5. Time-Awareness for Media-SynchronisationOpportunities \& Challenges 
6. NERstar: New Approaches to Improving the Quality of Audiovisual Media Services

7. Review of Media Sync Reference Models: Advances, Inconsistencies and Open Issues

The workshop includes as well five demos, one keynote, and interactive sessions. The demos are (apart from the ones showcasing paper 1 and 6):

- Merge and Forward - Self-Organized InterDestination Multimedia Synchronization

- Synchronization and Customization of Subtitles in Web-based Main-Screen and Multi-Screen Scenarios

- A customizable open-source framework for measuring and equalizing e2e delays in shared video watching

The draft schedule looks like this:

9.00 Welcome by the organizers

9:10 Keynote presentation

9.50 Short presentations

11.00 Break

11.15 Discussion about the topics and demos

12.30 Lunch

14.00 Work in subgroups
15.45 Short break

16.00 Presentations of the sub-groups, discussion

17.00 End

\section{Workshop Organization}

The workshop is organized by TNO, CWI and UPV. The main organizers are Hans Stokking (TNO), Pablo Cesar (CWI) Prof. Fernando Boronat (UPV), and Mario

Montagud (CWI). We would like to thank the Technical Program Committee that helps shaping the program of the workshop: Cyril Concolato, (Telecom ParisTech),

Davy Van Deursen (EVS), Luiz Fernando Gomes Soares (PUC-RIO), Jack Jansen (CWI), Tim Stevens (UK),

Christian Timmerer (Klagenfurt University), and Ishan Vaishnavi (Huawei)

\section{Bios}

Hans Stokking is a Senior Scientist and inventor at TNO in the field of Information and Communication Technology. His focus is on integrating various fields of study, and as such, Hans is active in the area of IPTV solutions, content distribution networks and home networks. His main focus is in 'how to get services across networks' and he has consulted a wide range of customers on a range of services and networks: from consumer and business VoIP services to Internet

services to P2P networks and inter-business messaging networks. Hans is (co-) author of over 20 popular and scientific papers, is (co-) inventor of more than 40 patent applications and has prepared many standardization contributions over the years. He is also a TPC member for the yearly ICIN conference, and is (co-) organizer of the international Media Synchronization Workshop series. 
Dr. Pablo Cesar leads the Distributed and Interactive Systems group at Centrum Wiskunde \& Informatica (CWI) in Amsterdam, the Netherlands. He obtained his PhD from the Helsinki University of Technology, in Finland, in 2005. He has participated and lead very successful EU-funded project like REVERIE, Vconect, TA2, iNEM4U, and SPICE. Pablo has (co)-authored over 70 articles about multimedia systems and infrastructures, social media sharing, interactive media, multimedia content modelling, and user interaction. Several of his publications have won the best paper award at a number of high-quality events such as ACM MMsys (2013), ACM HT (2011), EuroITV (2008) and ACM MM (2008). He has given tutorials about multimedia systems in prestigious conferences such as ACM Multimedia, $\mathrm{CHI}$, and the WWW conference. He is (co-) organizer of the international Media Synchronization Workshop series. Webpage: http://homepages.cwi.nl/ garcia/

Prof. Fernando Boronat studied Telecommunications Engineering at the Polytechnic University of Valencia (UPV), in Spain. After working for several Spanish Telecommunication Companies, in 1996 he moved back to the UPV, where he is an Assistant Professor in the Communications Department at the Gandia Campus. He obtained his PhD degree in 2004 and his main topics of interest are Multimedia Systems, Multimedia Protocols and Media Synchronization. He is IEEE Senior member (M'93, SM'11) and is involved in several IPCs of national and international journals and conferences. Webpage:

http://personales.upv.es/fboronat/Research/index_inve stig_en.html.
Dr. Mario Montagud (@mario_montagud) was born in Montitxelvo (Spain). He studied Telecommunications Engineering at UPV (Polytechnic University of Valencia), in Spain, and obtained the PhD degree from the same university in 2015. Since then, he is a postdoc researcher at CWI (The National Research Institute for Mathematics and Computer Science in the Netherlands). His topics of interest include Computer Networks, Interactive and Immersive Media, Synchronization and QoE (Quality of Experience). Mario is (co-) author of over 30 research and teaching publications, and has contributed to standardization with the IETF (Internet Engineering Task Force). He is also member of the Technical Committee of several international conferences and of the Editorial Board of international journals. Webpage: https:// sites.google.com/site/mamontor/

\section{Acknowledgments}

Fernando Boronat would like to thank the funding from the following project: GROUP_HYBSYNC (Hybrid and Inter-Destination (IDMS) Synchronization to enable enriched, personalized, inmersive and shared media experiences); MINECO, Ministerio de Economía y Competitividad of Spain (Ref. TEC2013-45492-R).

\section{References}

1] Media Synchronization Workshop series, see https://sites.google.com/site/mediasynchronization/

[2] Geek and Poke, see http://geek-andpoke.com/geekandpoke/2012/12/9/simply-

explained.html 